\title{
Describable but Not Predictable: Mathematical Modeling and Non-Naturalistic Causation
}

\author{
JONATHAN BARTLETT
}

The Blyth Institute

\begin{abstract}
Our notions of causation in science are often unintentionally constrained by the mathematics we use. Typically, scientific investigations use algebraic or calculus-based mathematics to model causes and effects. In these types of models, there is a predictive relationship between the cause and the effect. This predictive pattern is what most people use to classify events as materialistic, leaving events that are not so classified as non-materialistic. Mathematics over the last century has introduced new formalisms that cover functions that do not conform to the materialistic pattern. While these functions cannot always predict outcomes for typical cases, they can be studied and analyzed in other ways, and therefore can be used for knowledge-building. Therefore, by expanding the mathematical toolset, investigators can better identify and model non-materialistic causes.
\end{abstract}

\section{Introduction}

One of the largest barriers to considering alternatives to naturalism is that it is difficult for people to conceive of what sorts of causes lie outside of the naturalistic paradigm and how they might be modeled. While some people can see that there must be more to the world than what is contained in naturalism, most view those parts of reality as fundamentally enigmatic. This has even led many who disagree with naturalism on a fundamental basis to do their work under a naturalistic rubric. 
It has also prevented people from attempting rigorous studies of phenomena that are outside of naturalism's domain.

Therefore, for any alternative to methodological naturalism to take hold, methods of analyzing events that are beyond the reach of naturalism must be developed. Since non-naturalistic phenomena are categorically different than naturalistic phenomena, such methods will be necessarily different and may provide different kinds of information about the events than the kinds of information we are used to having. Such modeling should not be judged as successful or unsuccessful based on whether or not it matches the kind of information obtained from naturalistic models, but on whether or not it matches reality and provides helpful information that can inform decisions and can be combined with other forms of knowledge.

However, before we look at how to model non-naturalistic phenomena, we must first establish what it means for a particular phenomenon to be non-naturalistic.

\section{Computability as a Demarcation}

The first problem in developing alternatives to methodological naturalism is with determining what counts as a naturalistic or non-naturalistic phenomenon. While most people think that such a demarcation is intuitively obvious, on closer inspection, this becomes a rather difficult problem. Several attempts at creating a demarcation have been attempted and most of them have failed.

First of all, we will equate naturalism with physicalism - the idea that all knowable phenomena are in some sense physical. Without this restriction, naturalism just means "everything," and defining an idea as "everythingism" is unhelpful. Physicalism is what most people mean when they talk about naturalism. However, this leaves us to define what it means for something to be physical. Without a solid definition in place, the category is meaningless (Stoljar, 2009).

For instance, let us imagine that ghosts are real. Are they physical? By what criterion could we classify them either way? It is true that ghosts are often considered non-physical beings, but on what rule might we make such a determination?

Some might say that an event is physical if it is observable and detectable. Let's say, then, someone found a way to detect ghosts and their effects. Are ghosts then considered physical? A demarcation between physical and non-physical that puts ghosts on the "physical" side seems nonsensical. Others might say that an event is physical if it is testable. But, again, if we developed a mechanism that tests for ghosts, then ghosts would be physical.

Physicalists oftentimes define "physics" to include anything that has effects. However, non-physicalists believe that their categories (i.e., mind, spirit, etc.) do in fact have effects in the physical world. Therefore, not only is such a definition of physical nonsensical (given the ghost example), it also does not put itself at odds with the non-physicalist claims. Bartlett (2016) goes into more detail on the problems of 
demarcation for physicalism and materialism.

To make a real distinction between these classes of phenomena at all, we need an objective criterion that takes into account both the features that physicalists and non-physicalists find important. No criteria is likely to have universal agreement, but for a criteria to be worthwhile it should have at least some who agree with it on both sides of the issue.

One criteria proposed by physicalists to distinguish between physical and nonphysical phenomena is computability. Under this rubric, physical processes are those whose results can, at least in principle, be calculated by computational systems, while non-physical processes are those which cannot. This thesis is described and defended more fully in Bartlett (2014).

This demarcation has many advantages. First, it is objective. Computation and computability is a well-studied topic. One can prove that certain mathematical functions are non-computational. Second, it is used by physicalists themselves. Having a demarcation criterion that is agreed upon by both parties makes discussion and progress possible. Third, since computation is a finitary mode of acting, having computation as a criteria means that events themselves must obey finitary logic, which is a reasonable requirement from a physicalist standpoint.

Therefore, for this paper, we will use computability as the demarcation criteria between naturalistic and non-naturalistic modes of causation.

\section{Elementary Functions and Causation}

Many of our ideas of causation, especially in the sciences, come from our experiences in mathematical education. I have no general criticism of mathematical education to offer (I myself teach math in the way described here), but it is important to note that the way math is taught and the way we learn it predisposes us to thinking about reality in certain ways. When lecturing calculus-level students, I try to at least alert students to the ways that their experience with lower-level mathematics biases them to thinking about the world in certain ways.

Most of the functions familiar to students are what I will call well-behaved functions. The biggest defining character of smooth functions, at least as I am considering them, is that they are smooth; specifically, they are $C^{\omega}$ smooth over a majority of the important parts of their range. $C^{k}$ smoothness refers to the number of times that a function can be differentiated successfully resulting in a continuous function, with $k$ indicating the number of times a function can be differentiated.

For instance, take the following function:

$$
f(x)= \begin{cases}x^{2} & \text { if } x \geq 0 \\ 0 & \text { if } x<0\end{cases}
$$

This function has $C^{1}$ smoothness. The function itself is continuous, and its first 
derivative is continuous. However, its second derivative is discontinuous at $x=0$, because the left-hand limit is zero while the right-hand limit is two.

Polynomials by themselves are all $C^{\infty}$ smooth. Likewise, sin and $\cos$ are $C^{\infty}$ smooth. Most functions that students encounter in the early years of mathematics are $C^{\infty}$ smooth.

These functions are also $C^{\omega}$ smooth. This level of smoothness means that not only are the functions $C^{\infty}$ smooth, but also that their Taylor series expansion converges. This is hugely important in the natural sciences for a quite unexpected reason.

If a function is $C^{\omega}$ smooth, then that means a small sample of the data can be extrapolated out infinitely simply by observing the small sample with more and more detail. For example, to do a Taylor series for a function $f$ around a specific point $A$, I need only find the derivatives of the function near $A$ in order to model the entire function $f$. As I find the value of higher and higher derivatives of $f$ at point $A$, I get closer and closer to its behavior for any value at all.

The significance of this is that, for $C^{\omega}$ functions, to find the behavior of $f$ at $B$, for any $B$, I need only to analyze the behavior of $f$ at $A$ in sufficient detail. I have no need whatsoever of analyzing $f$ at $B$ or even near $B$ in order to have a reliable estimation of its value, assuming I can get enough resolution of the function $f$ at $A$.

For a more specific example, let's say that I had a phenomenon $P$ that followed the sin function such that the true effects of the phenomenon could be given by $P=\sin (q)$ (where $q$ is some other quantity that is affecting the output), but I was not aware of the existence of the sin function, nor of the fact that $P$ behaved that way. Let us also say that I could only vary $q$ within the range of 0 to 0.8 with my experimental apparatus, but could measure both $q$ and the result of the phenomena with infinite accuracy. Because the phenomena is $C^{\omega}$ smooth, this means that even with my limited data set, I can determine the behavior of $P$ for any value of $q$ using only this data.

So, if I vary $q$ between 0 and 0.8 I will get the graph shown in Figure 7.1

But, if I measure the velocities, accelerations, accelerations of accelerations, and so forth, I can eventually get the full picture of what the phenomena is doing in the long term, as you can see in Figure 7.2.

Therefore, even though my sample is monotonically increasing and has a range between 0 and 0.0998 (and is in fact nearly linear in this range), if I were able to analyze it with sufficient detail, I could calculate its true range (-1 to 1 ), and determine that it is cyclical with a period of $2 \pi$. So, even though I analyzed a near-linear, tiny portion of the function, by being able to view its velocities, accelerations, accelerations of accelerations, and so on to a sufficiently high resolution, I can determine the large-scale patterns of behavior of the function.

For functions that work this way, it is a fantastic quality. The problem, however, is that all of the functions we work with in lower-level mathematics have this quality, and therefore students make the assumption that functions should and must work 
Figure 7.1: The Sine Function Over a Restricted Domain

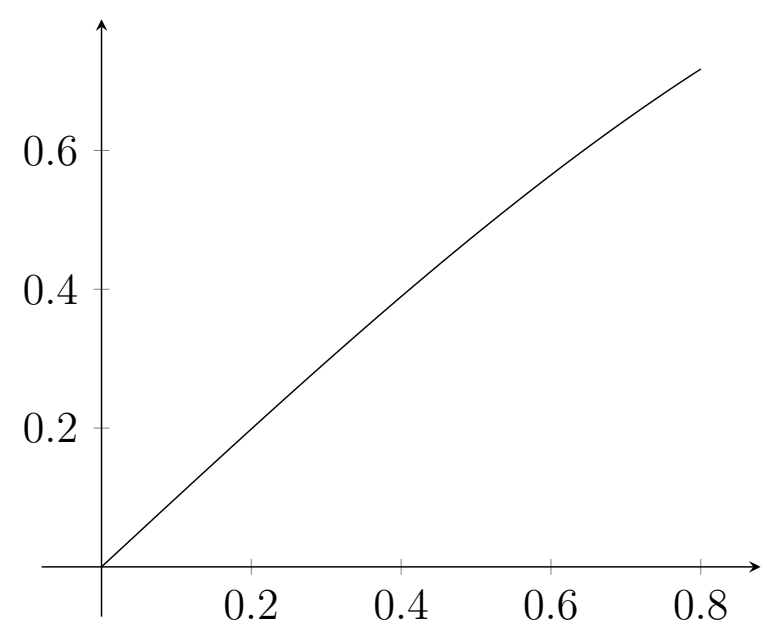

Figure 7.2: The Sine Function Over a Larger Domain

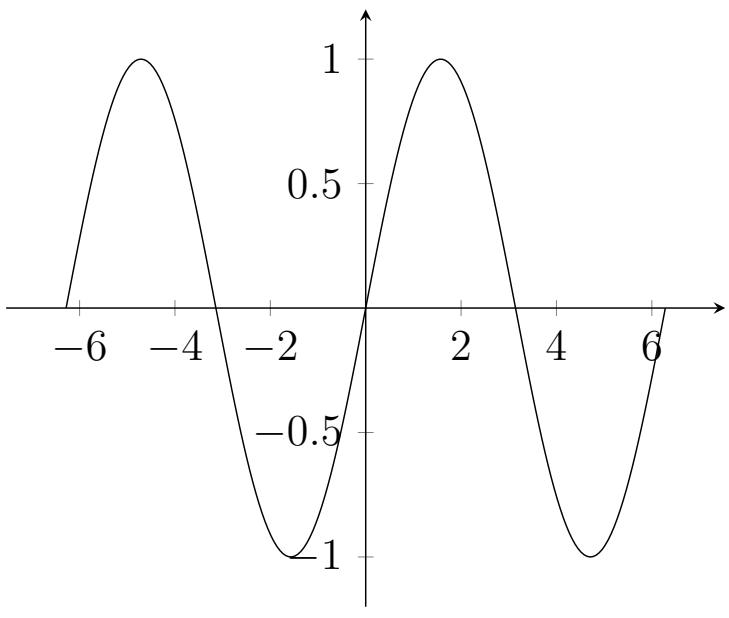


this way. The idea that there may be functions that do not behave this way is foreign to non-mathematicians. Therefore, when envisioning causation, it is assumed that causation itself must follow this pattern.

It may very well be true that causes that follow this pattern are easier to analyze using current scientific methodology. The question, though, is whether we want to limit science to only follow these causative patterns, or if we want to be able to find those that are more elusive.

As mathematical knowledge has progressed, more and more functions have been discovered that do not match traditional expectations in smoothness and other categories. However, their introduction into the sciences has been very slow due both to a lack of awareness and to metaphysical biases about whether or not causes can be modeled by them (van Rooij, 2008).

\section{Ways to Be Strange: A Partial Survey of Strange Functions}

In the previous section, we took a look at one way to be strange - by not being $C^{\omega}$ smooth. I should note that the purpose of the prior section was not to argue that all functions that are not $C^{\omega}$ are non-naturalistic, but rather to show how our expectations of how functions should behave intertwine with our expectations from nature, and how deviations from those expectation can affect both the way that we model nature and the way that we extrapolate our data to unknown values. In this section, we are going to look at what attributes of a function make it strange in a way that would lead us to consider that it is non-naturalistic.

As it happens, out of the total possible functions, most of them are not wellbehaved. That doesn't mean that realized functions are mostly not well-behaved, but the number of available pathological functions greatly outnumber those that are wellbehaved for almost any definition of pathological and well-behaved. Most of these functions at least appear useless and very likely are useless. However, there are several types of functions that are not well-behaved that may wind up being useful. Here we will do a short and incomplete survey of functions that may have the potential to model some interesting aspect of causation. We will term them "strange" rather than "pathological" to avoid the automatically negative connotations. The goal of this survey is not to say that any given function actually does model some event or process in real life, but to stimulate the imagination to help people think outside of traditional ways of thinking about causation and especially about modeling causation. 


\subsection{Primes, Number-Theoretic Functions, and Lengths of Causality}

Number theory has some of the easier-to-understand strange functions. Many of the functions in number theory are discrete rather than continuous so the question of smoothness doesn't really apply, though you can use analytic continuations to create real versions of some of them.

A simple example of a function from number theory is the prime number function, where Prime $(n)$ yields the $n$th prime number (starting with 2). For instance, Prime(5) would yield 11 because it is the 5th prime number.

Another interesting function from number theory is the distinct prime factors function, $\omega(n)$. (This is a different $\omega$ than the one for smoothness.) For this function, the result is the number of distinct prime factors for the given number. Therefore, $\omega(64)$ yields 1 , because 2 is the only prime factor, but $\omega(30)$ yields 3 because it has prime factors 2,3 , and 5 . Figure 7.3 shows a graph of this function.

What makes prime-oriented number-theoretic functions interesting is that the "length" of "causation" for each value varies. That is, the prime number 2 exhibits a causal relationship to every other number in the number chain, but the prime number 3 only exhibits a causal relationship to every third number in the chain. A prime number, then, is one in which there is no previous "cause" in play for its results.

Thus, while we normally think of causes as having uniform influences on future events as other causes, number-theoretic functions help us to think about nonuniform causation, where the reach of individual causes varies as well as the number of causative influences of any given event.

\subsection{The Cantor Function and Two-Way Causation}

A classic strange function is the Cantor Function. This function is strange because it is continuous over real numbers, almost entirely flat (i.e., non-flat spaces occur over a measure zero of the function space), and its $y$ value increases from 0 to 1 over the space of 0 to 1 on the $x$-axis. Wherever it is not flat, it has an undefined (i.e., infinite) derivative. The graph is given in Figure 7.4.

The graph of the Cantor function can be constructed as follows:

1. Start with a left-hand point at $(0,0)$ and a right-hand point at $(1,1)$.

2. Find the point exactly halfway between the left-hand point and the right-hand point, which we will call the midpoint. On the first iteration, this will be $\left(\frac{1}{2}, \frac{1}{2}\right)$.

3. Draw a horizontal line $\frac{1}{3}$ of the horizontal distance between the left-hand point and the right-hand point, centered on the midpoint, which we will call the midline. 
Figure 7.3: The $\omega(n)$ function from 0 to 100
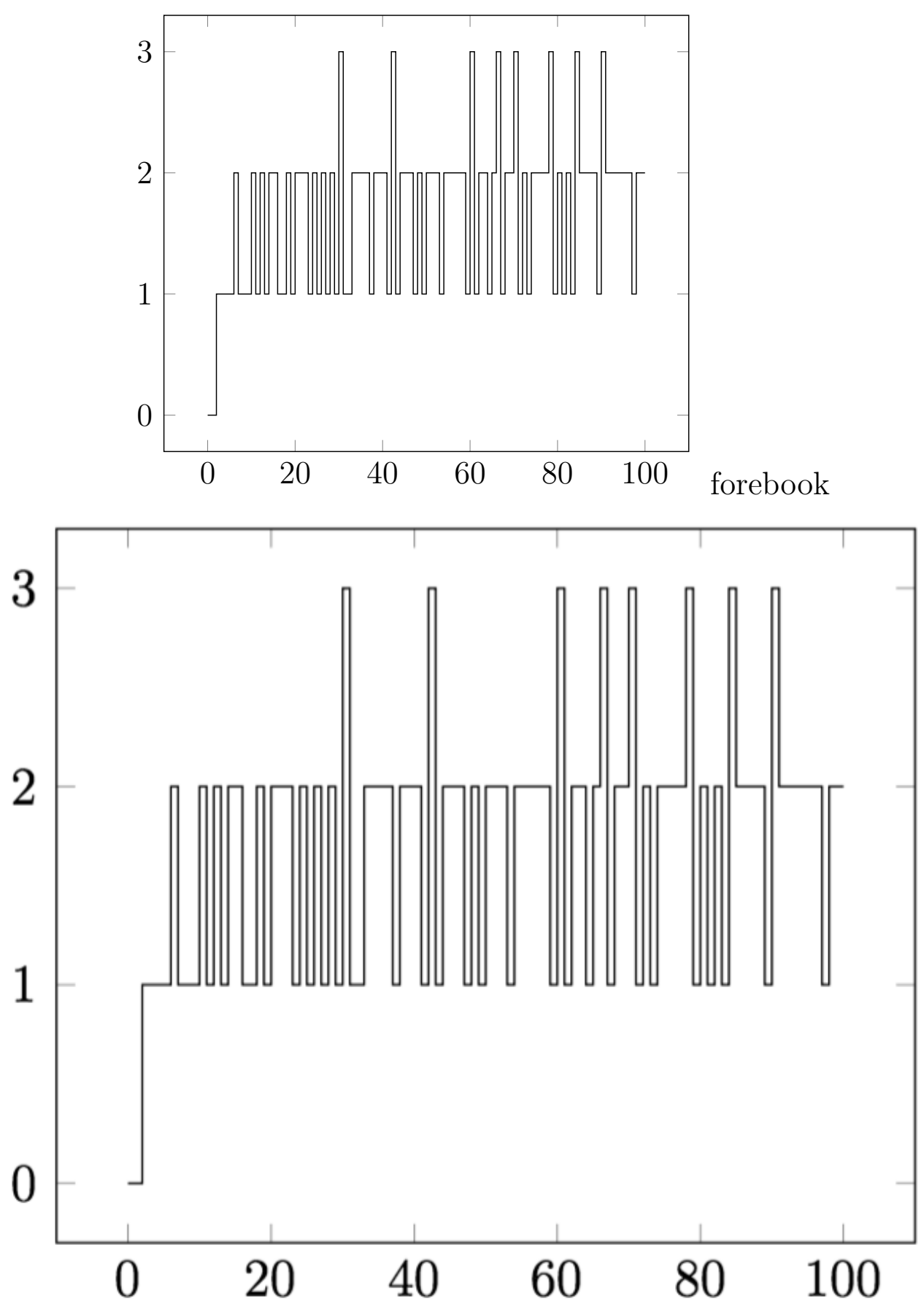
Figure 7.4: The Cantor Function on the Unit Interval

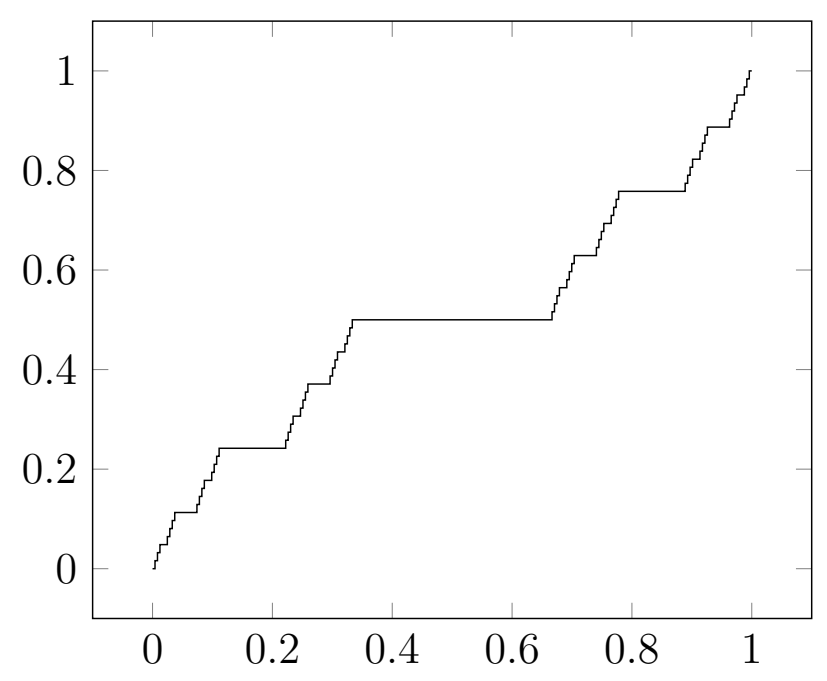

4. Perform steps 2-4 again twice. The first time we will use the current left-hand point as the new left-hand point and the leftmost point of the midline as the new right-hand point. The second time we will use the current right-hand point as the new right-hand point and the rightmost point of the midline as the new right-hand point.

This process will repeat indefinitely, with more and more intervals being created each time. Assuming it proceeds an infinite number of times, it will be a continuous function as described above.

What is interesting about the Cantor Function is that it describes two-way causation. That is, the function starts with left and right points, and the middle points are decided based on collaboration between them. Since many of the concepts of alternatives to methodological naturalism involve some sort of dualism between mechanical causes (where the initial conditions determine the outcome) and teleological causes (where the final desired outcomes determine the intermediary steps), this seems to be a place where a Cantor-like function may be beneficial.

Cantor-like functions establish a way to associate feedback between mechanical causes on the left-hand side and teleological causes on the right-hand side. While the Cantor Function itself may or may not model anything in particular in real life, this type of function can help us expand our reasoning capabilities to be able to model multidirectional causation.

\subsection{Assertion-Satisfying Functions}

In computer programming, Landin (1965) developed the concept of continuations, which is an abstract control structure, where your location in the program can be "bookmarked" to return to later. This allowed for the development of a variety 
of interesting control structures that work quite differently than the typical ones considered in computer programming (sequence, selection, and iteration).

One of the more interesting ones is the ability to develop assertion-satisfying functions. In an assertion-satisfying function, variables can take on ambiguous values (i.e., the variable $q$ could be assigned any of the values between one and five). Then, during execution, a particular value is tried, but if the results do not satisfy a downstream assertion, the results get thrown away, the function is backed up to the bookmark, and a new value for $q$ is tried. It is possible that reality works like this. It is possible that some of the laws of nature exist as assertions and that reality may be able to back up and "try again" if the assertions do not match. In such a scenario the laws of physics wouldn't so much dictate what must happen, but rather only the constraints of what could happen.

\subsection{The Halting Problem and Related Functions}

Another type of function that should be considered in modeling reality is the halting problem and related functions. This is described more fully in Bartlett (2014), but a condensed version will be provided here.

In computer programming, a computation is supposed to finish - it is supposed to complete and yield a result. This is known as "halting." Computations that do not finish are said to be caught in an "infinite loop." If you have ever had your computer stuck in a situation where the cursor just spins and spins and never stops, it is possible you have experienced an infinite loop. Computations are supposed to yield values, and when they don't, it leads to problems.

The deeper issue, however, is determining if a computation will halt or if it will get stuck in some sort of infinite loop. One of the first discoveries of computer science was the fact that there is no algorithmic way to tell if a given computation will halt or if it will go into an infinite loop. That is, I cannot write a program that will tell me, even if I know all of the inputs, whether or not a different program will yield a result or go into an infinite loop. This is known as the "halting problem" in computer science. For any given program/input combination, it will either halt or it won't - there is no other possibility. However, figuring out which ones won't halt is an impossible problem for a Turing-like machine.

There are many problems in computer science that are essentially incomputable but could be computed if we were counterfactually able to write a program that solved the halting problem. One such problem is the "busy beaver" problem. In the busy beaver problem, the goal is to find, for a program of length $N$, what the largest output a program can generate and still halt is. This is generally unsolvable, but could be easily solved if we were able to write a function to determine the result of the halting problem.

Even though we can't implement a function to tell us whether a function halts or not, we can reason about how such a function can be used and what sorts of 
properties it would have. An unimplementable function is called a Turing oracle or just an oracle. Thus even though we couldn't implement or predict the results of an oracle, those are not the only types of reasoning available. Alan Turing, for instance, used oracles to measure the relative difficulty of different types of problems, showing that some problems are more or less complex based on the kind of oracle required. Therefore, just because a function can't be explained in terms of its operation, this doesn't exclude it from useful reasoning or knowledge-building.

Additionally, as argued in Bartlett (2014), it appears that humans are able to solve something similar to the halting problem. If humans could not tell if a program would halt or not, they would not be able to successfully program computers. Therefore, it seems that humans have access to an oracle of some kind that allows them to solve problems that are beyond computation. Robertson (1999) points out that the development of mathematical axioms is itself a super-computational problem. Therefore, the ability of humans to develop mathematics itself shows that humans have access to some sort of Turing oracle.

Bartlett (2014) suggested that perhaps the oracle humans have access to is the ability to generate needed axioms based on existing problems. The oracle was

described as $A=I(Q, p, i, B)$, where $Q$ is the problem the human is attempting to solve (with inputs $p$ and $i$ ), and $I$ is the human "insight" oracle function that reveals the set of axioms, $A$, needed to solve the problem $Q$. The function requires that the human already have $B$ - the set of all axioms needed to solve the problem, except one. What is being proposed by the oracle function is that human insight is able to generate axioms (a non-computational event as described by Robertson (1999)) when humans are given a problem they cannot solve and all of the axioms they need to solve it, except one. Thus, even though this function isn't computable, it can be used to reason about non-mechanical models of the mind.

\section{Using Incomputable Functions in Modeling}

As we have seen, by expanding our view of mathematics beyond the typical well-behaved functions, we can incorporate models of non-mechanical (i.e., nonnaturalistic) causes and modes of operation into mathematical descriptions. Such mathematical descriptions would enable better integration between naturalistic and non-naturalistic causes at work in a system. For a system to be mathematical does not mean that it must be predictive or even computational. Many functions in mathematics are not computable, and in fact, computability may only cover a very small proportion of them.

One may wonder, why bother with mathematics at all? Mathematics is just a formalization of logic. Using mathematics requires that a person distill their ideas into the most rigorous and abstract form. Additionally, because mathematics as a discipline is well-studied, boiling ideas down into mathematical forms, even if incal- 
culable, allows mathematical tools to be used to analyze and reason from these ideas. It also makes it easier to combine different ideas. If each idea is expressed mathematically, then the combination of ideas can likely be expressed mathematically, and the logical consequences of these ideas can be more readily determined.

One of the advantages of our well-behaved functions is that their long-term behavior can be arbitrarily extrapolated from limited observations. Additionally, with a finite set of observations, it is difficult to distinguish a strange function from a more well-behaved nearby function, especially within the limited data set. A major issue with using strange functions in modeling is determining whether or not such a function is what is being observed or if its more well-behaved nearby function is at work.

Therefore, what is required for establishing a strange function as the basis for an observed effect is a logical reason for preferring the function. That is, there must be something in the nature of the causal relationships that would indicate the usage of a strange function in a model. One other thing that may indicate that a strange function is indeed required is the need to continually change the model with more and more data. This may indicate that a strange function is at play, and the causal relationships should be investigated to see if a strange function may properly model what is happening.

Since this paper only presents a small smattering of the known strange functions, more and more modeling power will be available by understanding more and more strange functions and how they relate to causality. The ones presented here were picked because the author could see ways in which they may be important to helping researchers think about causality. Additionally, more functions may be available by simple creative construction. Once the strictures of well-behaved functions are removed, and a person gets acquainted with the nature of strange functions, the ability to construct definitions of new strange functions to match the causality in question will be increased.

While strange functions should not be introduced lightly, there is no reason to avoid them in models. The preference for well-behaved functions is just that - a preference. There is no reason why reality must conform to our preferences. Linear components in models are better-behaved than non-linear components, but that doesn't mean our models must always contain linear components. The goal of modeling for science is to provide a deeper understanding of the nature of the subject under investigation. Other goals (such as using models for engineering) may substitute similar well-behaved functions in order to simplify calculations, but science, as an attempt to learn more about the true nature of reality, should in most cases prefer whatever function is the truest model of reality. 


\section{Testing Models that Use Strange Functions}

One of the key features of science is testability. As pointed out in Bartlett (2016), the two main features of methodological naturalism that made it successful were that it defined a scope of inquiry and it provided a system of justification. With wellbehaved functions, the system of justification is fairly straightforward. The model will predict how a system will behave for tests that have not been performed yet; the experimenter will then perform the tests and see if the results match the model within a margin of error. With strange functions, however, the models do not always predict behavior. Therefore, the system of justification used will have to be modified in order to accommodate strange functions.

However, before we look at how we can test our new models, we should think about why it is that we test models in the first place. The goal of testing is to allow reality to push back on our ideas. That is, we have ideas about the nature of reality, but our ideas must conform to external reality, not the other way around. Testing is done to make sure that reality has a chance to give us feedback on the truth of our ideas.

We should recognize that testing is not an absolute truth-teller. It is more of a sanity check than a rigid determiner of truth. For a finite set of data points, there are infinitely many functions that would be within the margin of error for those data points (Kukla, 1996). So how might someone decide between two empiricallyequivalent theories? As it stands, our scientific ideas do not emanate entirely from empiricism. If they did, then this would be a problem. Instead, empiricism provides the dataset that we use to establish rationalistic models, and it provides additional data to validate such models. But the models themselves are based on logical relationships between entities under investigation.

Therefore, the key to testing is not that the data points must be uniquely determined by the theory, but rather that the theory must flow from a proposed logical relationship between entities and the data must be consistent with it within a margin of error.

Even though strange functions are not always calculable or predictive, they do lend themselves to reasoning about relationships, otherwise they would not be considered functions. Therefore, it is possible to find patterns that are true with a strange function that may be tested for, even if the strange function itself is not directly testable. In the next section, we will look at a specific example.

\section{Randomness as an Exemplar Strange Function}

While strange functions generally have not been given much scientific weight, one in particular has been used regularly - randomness. While randomness is not wellbehaved like most of the functions within science, the willingness of scientists oper- 
ating under methodological naturalism to use it probably stems from the fact that it does not appear on the surface to imply teleology (though see Bartlett (2008) for an alternative view). Therefore, its adoption in the scientific community as a viable model allows us to demonstrate the utility of strange functions in scientific modeling.

Randomness is actually a property of an infinite sequence. Therefore, no finite sequence of events can prove that the sequence is random. Additionally, randomness is not predictive. Therefore, including randomness in the model does not help to predict any specific outcome. Thus, randomness matches what we have called strange functions.

Since randomness doesn't predict a specific outcome and cannot be tested directly, how was it included in scientific theories? Basically, if a model of an event has multiple possible outcomes, and the outcomes proceed in an order that is statistically stable but does not point toward any other structure, then the suggestion of randomness is quite appropriate.

For instance, in a Poisson distribution, the mean is equal to the variance. Therefore, one can "test" for such a distribution by checking the mean against the variance. If they are close, then the suggestion that the process is a random process following a Poisson distribution can be maintained. There are many different ways that the mean can equal the variance, but if our formal reasoning leads us to expect such a distribution, and the distribution's characteristic features match our expectations, then the test can be considered confirmed. This is used, for instance, in the Luria-Delbrück experiment where the test for randomness is used to determine if a mutation is in response to a selective pressure or if the mutation preceded the selection.

\section{Conclusion}

The goal of the present paper is not to propose a specific idea or procedure, but rather to assist researchers proceeding in directions at odds with methodological naturalism by pointing to the stranger aspects of mathematics that can serve as tools when investigating non-naturalistic phenomena. My hope is that researchers will be able to unshackle their imaginations from the mathematics of naturalism but without losing the rigor necessary to develop well-founded theories of how different aspects of the world works. Likewise, making use of mathematics even when it isn't well-behaved will better enable integration of different models and phenomena. 


\section{References}

Bartlett, J. 2008. Statistical and philosophical notions of randomness in creation biology. Creation Research Society Quarterly 45:91-99.

Bartlett, J. 2014. Using Turing oracles in cognitive models of problem-solving. In J. Bartlett, D. Halsmer, and M.R. Hall (editors), Engineering and the Ultimate, pp. 99-122, Blyth Institute Press, Broken Arrow, OK.

Bartlett, J. 2016. Philosophical shortcomings of methodological naturalism and the path forward. In J. Bartlett and E. Holloway (editors), Naturalism and Its Alternatives in Scientific Methodologies, Blyth Institue Press, Broken Arrow.

Kukla, A. 1996. Does every theory have empirically equivalent rivals? Erkenntnis 44:137-166.

Landin, P.J. 1965. A correspondence between algol 60 and church's lambda-notation: Part i. Communications of the ACM 8(2).

Robertson, D.S. 1999. Algorithmic information theory, free will, and the Turing test. Complexity 4(3):25-34. http://cires.colorado.edu/ doug/philosophy/info8.pdf

Stoljar, D. 2009. Physicalism. In E. Zalta (editor), The Stanford Encyclopedia of Philosophy, The Metaphysics Research Lab, fall 2009 edition. http://plato.stanford.edu/archives/fall2009/entries/physicalism/

van Rooij, I. 2008. The tractable cognition thesis. Cognitive Science: A Multidisciplinary Journal 32(6).

http://staff.science.uva.nl/ szymanik/papers/TractableCognition.pdf 